Physics

Physics Research Publications

Purdue University

Year 2003

\title{
High-speed wide-field coherence-gated imaging via photorefractive holography with photorefractive multiple quantum well devices
}
C. Dunsby*
D. Mayorga-Cruz ${ }^{\dagger}$
I. Munro
Y. $\mathrm{Gu}^{* *}$
P. M. W. French ${ }^{\dagger \dagger}$
D. D. Nolte $\mathrm{e}^{\ddagger \ddagger}$
M. R. Melloch ${ }^{\S}$

$*$
$\dagger$
$\ddagger$
$* *$
$\dagger \dagger$
$\ddagger \ddagger$
$\S$

This paper is posted at Purdue e-Pubs.

http://docs.lib.purdue.edu/physics_articles/420 


\title{
High-speed wide-field coherence-gated imaging via photorefractive holography with photorefractive multiple quantum well devices
}

\author{
C Dunsby ${ }^{1}$, D Mayorga-Cruz ${ }^{1}$, I Munro ${ }^{1}, \mathbf{Y ~ G u}^{1}, \mathbf{P}$ M W French ${ }^{1}$, \\ D D Nolte ${ }^{2}$ and M R Melloch ${ }^{2}$ \\ ${ }^{1}$ Photonics group, Physics Department, Imperial College London, Prince Consort Road, \\ London SW7 2BW, UK \\ 2 Department of Physics, Purdue University, West Lafayette, IN 47907, USA
}

Received 4 June 2003, accepted for publication 26 June 2003

Published 27 October 2003

Online at stacks.iop.org/JOptA/5/S448

\begin{abstract}
The parallel pixel acquisition inherent in wide-field coherence-gated imaging techniques offers the possibility of high-frame-rate imaging and volumetric imaging, including through scattering media. We discuss photorefractive holography using sensitive, dynamic photorefractive multiple quantum well (PRQW) films as a promising approach and, after reviewing the factors that determine the sensitivity of this technique, we demonstrate that high-frame-rate wide-field coherence gated imaging is possible at 830 frames per second with PRQW devices using a non-intensified camera to record the diffracted signal.
\end{abstract}

Keywords: High-speed, photorefractive, holography, coherence gating, wide-field

\section{Introduction}

High-resolution imaging through scattering media using nonionizing optical radiation has many potential applications for imaging through a range of scattering media including fog, seawater and particularly biological tissue. In order to minimize absorption when imaging in tissue, it is possible to use near-infrared radiation that corresponds to a minimum in the tissue absorption spectrum. In this spectral region, it is optical scattering that presents the greatest obstacle to high-resolution imaging, as the exponential attenuation of the least scattered 'ballistic' light due to scattering is much greater than the attenuation due to absorption. For sufficiently thin scattering samples, there will be some unscattered photons emerging from the sample and, when imaging in transmission, these first-arriving, least-scattered photons may be preferentially selected by applying an appropriate timegating mechanism to provide high-resolution images. For reflection imaging geometries, the application of a time gate provides depth-resolved (time-of-flight) imaging and also rejects much of the scattered light, although there may be crosstalk with light scattered from different depths in the sample.

Generally, techniques for selecting ballistic photons emerging from a sample may be divided into incoherent and coherent gating methods [1]. Incoherent time-gating techniques combine short optical pulses with either a fast electronically controlled shuttering device or exploit a nonlinear optical effect to realize an ultrafast shutter. Coherence gating techniques utilize time-of-flight gated interferometric detection with short coherence length light that is not necessarily of short temporal duration. An interference signal is only detected when the sample and reference arms of the interferometer are matched to within the (short) coherence length of the source and so it is possible to determine the reflectivity of the sample at a particular depth whilst rejecting light that has travelled different pathlengths. Thus, the coherence length of the source determines the depth resolution. The most mature coherence-gated imaging modality is optical coherence tomography (OCT) [2], which is commonly implemented using a spatially coherent, short coherence-length source such as a mode-locked laser or 
superluminescent diode (SLD), which is coupled into a fibrebased Michelson interferometer. OCT achieves very high dynamic range detection through the combined use of lock-in detection and high dynamic range single-channel photodiode detectors. Additionally, the use of a single-mode fibre interferometer provides a confocal spatial filter, which can reject much of the unwanted scattered light, depending on the numerical aperture of the optical system. However, OCT is a single-pixel detection scanning technique and, as well as scanning the reference pathlength $(z)$, must also scan laterally $(x, y)$ to create 2D $(x-z)$ or 3D $(x-y-z)$ images. The requirement for two-dimensional scanning does limit the achievable frame rate, although video rate OCT has been achieved using relatively high power optical sources, e.g. [3] The requirement for high average power sources with high spatial and low temporal coherence is normally met by modelocked solid-state lasers and this makes high-speed OCT instrumentation relatively complex and expensive.

It is therefore interesting to investigate wide-field techniques that provide parallel-pixel acquisition with reduced scanning requirements and are able to take advantage of relatively simple and low-cost broadband optical sources of low temporal and spatial coherence, such as LEDs or thermal sources, e.g. [4]. These approaches may be considered in three categories, according to whether they utilize interference in either the temporal, spectral or spatial domains. OCT records a time varying interference signal, and this may be extended to wide-field $(x-y)$ detection through the use of a detector array (e.g. a CCD camera) and multiple image acquisitions. However, CCD frame rates are much slower than the response times of photodiodes and therefore either phase-stepping of the reference arm [5] or phase-shifting in combination with stroboscopic illumination [6] must be used to allow the coherent image to be extracted, typically by sampling the interference signal at four different relative phases between the sample and reference arms. Using broadband thermal light sources to acquire images at a series of depths has provided 3D images of biological samples with approximately micrometre resolution in all three spatial dimensions [4]. This approach requires that the sample remain interferometrically stable over the four acquisitions, which may limit the ability to image moving samples, although this may be addressed by acquiring all four phase-stepped images simultaneously [7]. Wide-field coherence-gated imaging suffers from the fact that both the coherent signal and the background of scattered light are incident on the (CCD) detector and so it is often necessary to employ averaging to enhance the dynamic range such that the weak interference signal can be detected above the shot noise. Spatial filtering may also be employed to reduce the background of the scattered light. Another temporal interference approach is that of smart pixel arrays [8], which provide on-chip band-pass and amplification electronics for each pixel of a $58 \times 58$ array, i.e. effectively implementing parallel OCT. The frequency domain approaches, sometimes described as spectral interferometry, can probe an entire depth scan at a particular point on the surface of the sample in one acquisition using a linear detector array [9] or instead capture an entire $x-z$ slice simultaneously if a 2D detector array is used [10]. Again, the dynamic range may be improved through the use of averaging, provided that the sample is interferometrically stable over the total acquisition time. The spatial domain approaches utilize spatial interference patterns - or holograms - from which the coherent images are extracted. Holographic imaging as a means of imaging in the presence of a scattering medium was first proposed by Stetson [11] and later improved by Caulfield [12]. These techniques used photographic film as the recording medium and so real-time imaging was not possible; recent work has overcome this limitation through a variety of methods including the direct recording of holograms by a CCD $[13,14]$ and photorefractive holography using barium titanate $[15,16]$ and photorefractive multiple quantum well (PRQW) devices [17].

As mentioned above, wide-field coherence-gated imaging techniques can take advantage of the relatively low-cost, highpower sources of low temporal and spatial coherence. In practice the use of sources of low spatial coherence is highly desirable because this reduces interpixel cross-talk that occurs if photons are scattered from their original paths but arrive at adjacent pixels with the appropriate pathlength to interfere with the reference beam-thus introducing speckle noise. By making adjacent pixels of the image incoherent with one another (i.e. making the detector pixel spacing or the effective source size sufficiently large) this effect can be reduced [18] and the interpixel interference averages to a dc background. Sun et al [19] have shown that image plane holography with spatially incoherent sources is equivalent to confocal imaging. This dc background presents a problem however, since it contributes to saturation of the detector (CCD) - as does any incoherent scattered light. Thus, compared to OCT, there is more unwanted (scattered) light reaching the detector and also the dynamic range of a CCD is significantly lower than that of the photodiodes used in OCT. Wide-field coherencegated techniques therefore suffer from a much lower effective dynamic range when imaging through scattering media and their penetration depth is probably less than that achievable with OCT. This disadvantage is offset to some extent by the observation that, when imaging through a significant number of scattering mean free paths but at sample depths for which ballistic photons are still detected, the OCT signal can be degraded by 'axial speckle'. This results from interference of photons that multiply scatter, probing different regions of the sample but recovering their original trajectories such that they are detected through the confocal spatial filter.

Ideally, wide-field coherence-gated techniques require an imaging detector with very high dynamic range-or one which is insensitive to the background of scattered light. The most commonly used detectors are CCD cameras, where the dynamic range for the detection of small changes in intensity is limited by the shot noise. If the CCD is operated close to saturation then the shot noise can be determined by the full-well capacity of the pixels. For a typical scientific CCD camera with a full-well capacity of $10^{5}$, the smallest detectable change in intensity is $\sim\left(10^{5}\right)^{-1 / 2}$. In order to increase the dynamic range, it is necessary to use sensors with much larger well capacities - which is technologically challenging and expensive-or to employ averaging. Either approach leads to an increase in the image acquisition time if the incident intensity remains unchanged. An alternative approach is to develop imaging sensors that are insensitive to the 
scattered light background and this is the opportunity offered by photorefractive holography, which responds not to the integrated incident intensity but to its spatial derivative. This paper aims to review photorefractive holography, particularly using PRQW devices, and outline the factors affecting the performance of this approach. One such factor is the optical quality of the photorefractive medium, because unwanted scattering of the readout beam by heterogeneities in the medium becomes the dominant source of noise that determines the achievable dynamic range. The performance of a typical, but not optimized, PRQW device is presented here, including a demonstration of what is, to the best of our knowledge, the fastest depth-resolved imaging rate yet achieved, at 830 frames per second (fps).

\section{Photorefractive holography}

Photorefractive materials differ from conventional detectors as they respond to spatial variations in the incident illumination rather than the absolute intensity. A spatially modulated distribution of photons (e.g. a fringe pattern) incident on a photorefractive medium will generate a spatial distribution of charge carriers and then material dependent mechanisms for carrier transport and trapping give rise to a spatial modulation of the internal electric field that has the same distribution, apart from a possible phase shift, as the incident illumination. This spatial variation in internal electric field is converted to a spatial distribution of refractive index and/or absorption variations of the material through its electro-optical properties. It is usually possible to record a hologram within a photorefractive medium and then reconstruct the recorded hologram in real time, since there is no requirement for the hologram to be chemically developed (as is necessary with photographic film). Because an incident light field of uniform intensity will not produce a spatially modulated distribution of charge carriers, it will not be recorded by the photorefractive effect. Thus a recorded hologram corresponds to only those components of an object beam that interfere with the reference beam to produce a fringe pattern and so any uniform (dc) scattered light components are rejected. For an ideal photorefractive medium, the readout beam is diffracted by the recorded fringe pattern such that the reconstructed beam contains only the desired coherent image, which may be recorded by a detector (e.g. CCD)—essentially in a background-free manner. If low-coherence radiation is used, photorefractive holography thus provides a high-speed wide-field 3D imaging technique that is insensitive to scattered light.

Low-coherence photorefractive holography has previously been demonstrated with both rhodium-doped barium titanate $\left(\mathrm{Rh}: \mathrm{BaTiO}_{3}\right)$ crystals and AlGaAs/GaAs PRQW devices. In the case of $\mathrm{Rh}: \mathrm{BaTiO}_{3}$ the space charge field is converted into a variation in absorption/refractive index via the linear Pockel effect. Rh:BaTiO 3 can be grown in relatively large crystals having high optical quality that exhibit large diffraction efficiencies. These factors permit the recording of holograms in the presence of large incoherent backgrounds [20]. Unfortunately, however, the response time of this material can be of the order of seconds, depending on the incident intensity and wavelength, and so it is not suitable for real-time holographic recording and reconstruction. In comparison, thin semiconductor PRQW devices offer extremely fast grating buildup times and require very low incident intensities in order to saturate the photorefractive response. In these devices, the electro-optic properties are enhanced by operating near to the exciton optical resonance. The exciton response remains significant at room temperature due to confinement within the quantum well potentials, which typically consist of 100 alternating layers of GaAs wells and AlGaAs barriers. PRQW devices may be operated with an electric field applied either in the plane of the device or perpendicular to the device. When operated with an electric field applied parallel to the plane of the device, electroabsorption and associated electrorefraction are generated through electric field induced broadening of the exciton transition. This is termed the transverse Franz-Keldysh geometry. For an electric field applied perpendicular to the device, it is an electric field induced shift in the exciton transition energy (quantum confined Stark effect) that produces the electroabsorption and electrorefraction.

The PRQW devices used in this work are operated in the transverse Franz-Keldysh geometry. Values for the PRQW input diffraction efficiency (ratio of diffracted to incident intensities), $\eta_{\mathrm{p}}$, can reach $4 \times 10^{-3}$ for an applied electric field of $10 \mathrm{kV} \mathrm{cm}^{-1}$. Values for the grating saturation intensity (occurring when the photoconductivity exceeds the dark conductivity), $I_{\text {sat }}$, can be as low as $20 \mu \mathrm{W} \mathrm{cm}^{-2}$ for an applied electric field of $5 \mathrm{kV} \mathrm{cm}^{-1}$ [21]. In general, the high absorption, low saturation intensity and fast response times make PRQW devices the most sensitive photorefractive media for real-time (and faster) imaging applications. The work presented here uses AlGaAs/GaAs PRQW devices in an experimental configuration equivalent to non-degenerate four-wave mixing [22], i.e. with separate writing and readout beams. The electrorefraction and electroabsorption are strongly wavelength-dependent around the exciton absorption peak and therefore it is necessary for the readout beam to be tunable so that the maximum diffraction efficiency can be attained. Generally, the peak diffraction efficiency corresponds to a peak of the electrorefraction. The use of a separate writing and readout beams means that there are no wavelength or spatial coherence requirements for the write beam, other than the necessity of exciting above the band edge at around $840 \mathrm{~nm}$. This permits the use of many broadband optical sources suitable for coherence-gated imaging. Imaging in an alternative geometry, equivalent to that used for degenerate four-wave mixing, may also be successfully employed [23]. The effective efficiency of the photorefractive holographic detection has previously been investigated [24] and has been shown to be approximately equal to half the input diffraction efficiency of the device.

At first sight, the insensitivity of the photorefractive effect to the scattered light background would seem to make this approach compare very favourably to direct hologram detection using a CCD. The use of a readout beam at a different wavelength from the imaging radiation (i.e. the writing beams) means that all photons scattered by the sample can be prevented from reaching the CCD camera. In practice the dynamic range of this technique is compromised, not by light scattered in the sample, but by readout beam photons that are scattered by imperfections in the photorefractive medium. This varies 
according to the medium and the growth/fabrication techniques but has so far proved a significant issue. Our PRQW devices are grown on a GaAs substrate that is opaque to wavelengths near to the exciton response and so it is necessary for the substrate to be removed after growth. The first step towards removing the substrate is to attach the top of the PRQW device to a glass substrate using epoxy resin. The GaAs substrate can then be removed through a careful sequence of mechanical lapping and chemical etching processes. During the attachment of the PRQW device to the glass, some small air bubbles can become trapped in the epoxy and additional, much smaller defects may also be introduced in the etching and growth processes. These various defects limit the performance of the present generation of PRQW devices. In future, however, commercial clean room fabrication techniques will significantly reduce or even eliminate many of these scattering imperfections. The impact of PRQW device imperfections is discussed further in section 4.

\section{Experimental set-up}

The experimental system typically comprises an imaging interferometer with the PRQW device placed at an image plane where an image plane hologram is formed, as indicated in figure 1(a). A lateral fringe pattern is introduced by tilting the reference arm mirror and a polarization splitting interferometer is utilized to allow the easy adjustment of the relative intensity ratio between the object and reference beams. This image plane recording geometry is necessary when using spatially incoherent writing beam sources. For this particular set-up, the magnification of the object onto the PRQW device aperture was approximately unity, giving a field of view equal to the width of the device $(\sim 2 \mathrm{~mm})$. Previously, Linnik type geometries with greater magnifications have also been used [25].

For convenience, the broadband source used for this work was a (spatially coherent) tunable broadband diode-pumped cw Cr:LiSAF laser operated at $825 \mathrm{~nm}$ that provides spectral widths up to $\sim 20 \mathrm{~nm}$ and $\mathrm{cw}$ output powers approaching $100 \mathrm{~mW}$ when pumped with a $500 \mathrm{~mW} 100 \mu \mathrm{m}$ stripewidth red diode laser [26]. The readout beam was provided by an external cavity laser diode (ECLD) that was coupled into a single-mode fibre for convenient delivery and to yield a spatially uniform output beam. For our current device the peak diffraction efficiency occurs for a readout beam wavelength of $831 \mathrm{~nm}$. The readout beam is incident at an angle of approximately $30^{\circ}$ to the normal of the PRQW and the diffracted signal is selected by means of a Fourier plane spatial filter, S1. The position and angular extent of this aperture is an important factor in determining the performance of the system. Figure 1(b) shows a typical reconstructed holographic image of a USAF test chart. The bright spots result from scattering of the readout beam by bubbles in the epoxy layer, as discussed in section 2. Figure 1(c) shows a background subtracted imagethe background being recorded with the interferometer arms mismatched by more than the coherence length.

In order to produce the spatial fringe pattern it is necessary to introduce a tilt of the reference arm mirror. However, this is not ideal if short coherence lengths or small fringe periods are to be used as this tilt causes the pathlength difference between the object and reference beams to vary across the (a)

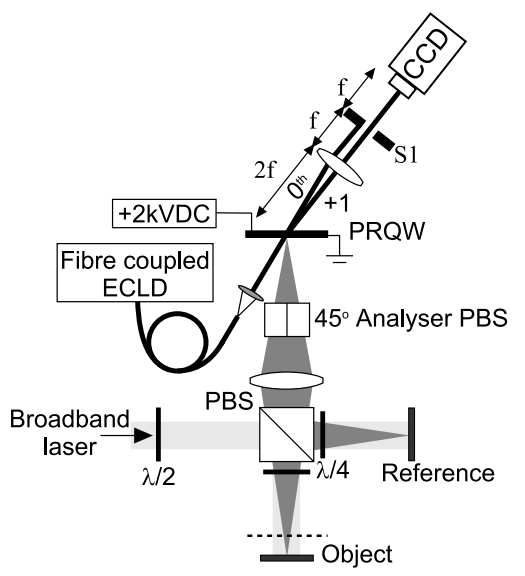

(b)

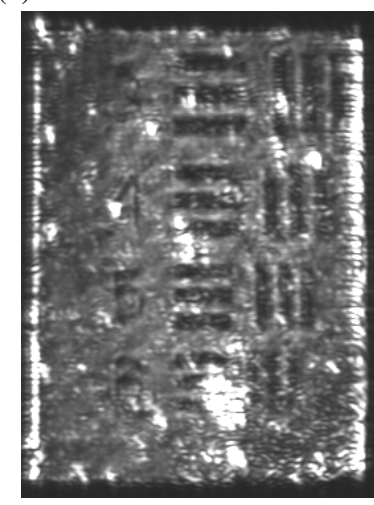

(c)

Figure 1. (a) Experimental set-up. PBS polarizing beam splitter cube, $\mathrm{S} 1$ slit, $\lambda / 2$ and $\lambda / 4$ half and quarter wave plates respectively. ECLD, external cavity laser diode. (b) Image of reconstructed hologram of a USAF test chart including background of scattered light from PRQW defects. (c) Background subtracted image. Both (b) and (c) are displayed using the same grey scale.

PRQW aperture and this can lead to a reduction in the field of view. This effect can be removed through the use of achromatic diffraction-grating based interferometers [27, 28]. However, these methods often suffer from low optical efficiencies unless high diffraction efficiency phase-grating beam splitters are employed. Another technique utilizes prisms in the interferometer close to the image plane, such that the energy fronts are tilted with respect to the phase fronts so as to achieve a high fringe modulation depth across the whole field of view [29].

\section{Factors determining the performance of image plane holography with photorefractive media}

\subsection{General considerations}

There are a number of important parameters that must be considered when performing image plane holography with photorefractive media, of which the most fundamental is the spatial frequency of the interference fringe pattern, $u$, which is determined by the angle that the reference beam makes with the object beam. The input diffraction efficiency of the photorefractive medium, $\eta_{\mathrm{p}}(u)$, is defined as the ratio of the diffracted to incident beam intensities and this depends on the spatial frequency of the fringe pattern through the processes of 
carrier drift and diffusion within the photorefractive medium. While it is desirable to set the spatial frequency of the interference fringe pattern such that the diffraction efficiency is maximized, there are other issues that must also be taken into consideration when optimizing this parameter.

As discussed above, the major source of noise in image plane photorefractive holography is that of unwanted scattering from imperfections in the photorefractive medium [24]. Any imperfections in a photorefractive medium will scatter unwanted readout beam light into the same direction as the desired diffracted signal and this will appear as an unwanted background at the readout CCD camera that must be subtracted (e.g. see figure 1(b)). The ability of the readout CCD to successfully subtract this background is determined by the full-well capacity (assuming that the CCD is operated near to saturation). In order to analyse the amount of light scattered by these imperfections, it is possible to introduce a scattering efficiency $\eta_{\text {scat }}$ (ratio of scattered to incident intensities) that is a function of the angle of scattering and the solid angle subtended by the detector. As can be seen in figure 1(a), a Fourier plane spatial filter is used to select the diffracted readout image and this aperture defines both the lateral resolution of the readout image and the amount of scattered light reaching the detector (since this aperture controls the effective solid angle subtended by the detector).

Therefore, when performing image plane holography there are a number of (conflicting) requirements that must be satisfied when choosing the spatial fringe frequency and Fourier aperture size:

- Diffraction efficiency-larger diffraction efficiencies correspond to shorter detector integration times and therefore higher potential frame rates.

- Lateral spatial resolution of final image-high-resolution images require a high spatial frequency fringe pattern and large aperture at $\mathrm{S} 1$.

- Walk-off-if no walk-off compensation scheme is used then high spatial frequency fringes are not compatible with short coherence length light.

- Signal to noise ratio-when imaging weak coherent signals, it is important that a small Fourier plane aperture is used so as to minimize the amount of light scattered from imperfections in the photorefractive that reaches the detector.

In order to fully optimize the optical set-up in view of these trade-offs, it is necessary to consider how the diffraction efficiency and the amount of unwanted scattering from imperfections vary as a function of the fringe spatial frequency.

\subsection{Measurement of scattering from $P R Q W$ defects in the Fourier plane}

In [24] we investigated the amount of parasitic light scattering per unit solid angle from a PRQW device using a set-up equivalent to that shown in figure 1(a). The amount of diffracted signal detected at the CCD was measured for a particular region of a PRQW device exhibiting high optical quality, and compared to the scattered light signal from the same region. The effective solid angle subtended by the detector was $5 \times 10^{-4}$ sr and the maximum value of $\eta_{\mathrm{p}} / \eta_{\text {scat }}$ was found to be $\approx 100$. However, in this previous investigation, the variation in $\eta_{\mathrm{p}} / \eta_{\text {scat }}$ as a function of the diffraction angle was assumed to be small for commonly used fringe spatial frequencies. While this is a useful approximation for homogeneous photorefractive media that is supported by the results presented below, it does not take into account the angular distribution of the background of scattered light due to the PRQW device imperfections or the variation in $\eta_{\mathrm{p}}$ as a function of the angle of diffraction.

The aim of this section is to describe the measurement of the scattering efficiency as a function of angle of deviation of the scattered light and to compare it to the diffraction efficiency at the same angle. More specifically, the procedure described below provides a direct method for measuring the output diffraction efficiency of the PRQW device (ratio of diffracted to undiffracted beam intensities) and simultaneously recording the angular distribution of the unwanted scattered light. One important point to note is that this measurement is based on the angular distribution of light scattered averaged over the whole PRQW device aperture, whereas our previous work only considered light scattered from a small region of the device.

The spatial frequency of the illuminating fringe pattern, $u$, determines the angle of the diffracted signal, $\theta$, as well as maximum achievable diffraction efficiency, $\eta_{\mathrm{p}}(u)$. The spatial frequency of the fringes and the angle of diffraction are related by

$$
\sin \theta=\lambda u=\frac{\lambda}{\Lambda}
$$

where $\lambda$ is the wavelength of the readout beam and $\Lambda$ is the period of the interference pattern. The parameter $\eta_{\text {scat }}$ is a function of both $\theta$ and the effective solid angle subtended by the detector, $\Omega$. It is convenient to consider $\eta_{\text {scat }}(u, w)$, where $w$ is the width of a square plane aperture (in units of spatial frequency) at a Fourier plane, e.g. the plane of slit S1 in figure 1. Note that the maximum spatial frequency and hence maximum resolution in the reconstructed image is then equal to $w$.

To measure $\eta_{\mathrm{p}}(u) / \eta_{\text {scat }}(u, w)$, the PRQW was illuminated with a sinusoidal interference pattern with a spatial frequency of 30 lines $\mathrm{mm}^{-1}$. The readout beam was then diffracted through the photorefractive grating and a video CCD camera (Pulnix PE2015) was placed in the Fourier plane of S1. In order to increase the dynamic range of the measurement, two sets of data $(1,2)$ were recorded with different $(0.1$ and $20 \mathrm{~ms}$ ) exposure times and then combined to produce the data presented here. For the $20 \mathrm{~ms}$ exposure acquisition, the bright undiffracted zeroth order peak saturated the CCD pixels but, away from this peak, the weak scattered light could be observed above the background noise level. For each exposure time, an image of the Fourier plane diffracted/scattered light distribution (a) and two dark background images (b, c), taken with both the writing and readout beams blocked, were recorded. These two background images were differenced $(b-c)$ to give a measurement of the background noise fluctuations. Each acquisition was averaged over 2000 video frames to reduce shot noise.

Figure 2(a) shows the image obtained for the $20 \mathrm{~ms}$ exposure by subtracting the corresponding dark background acquisition from the image of the diffracted/scattered light 
High-speed photorefractive holography with PRQW devices

(a)

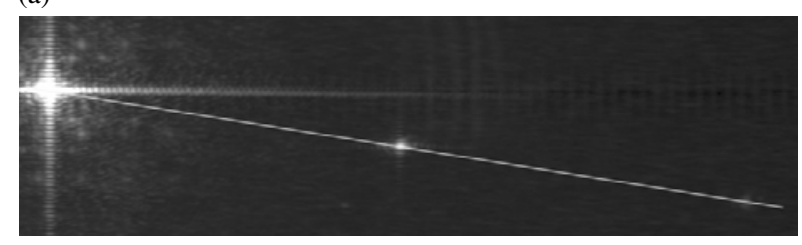

(b)

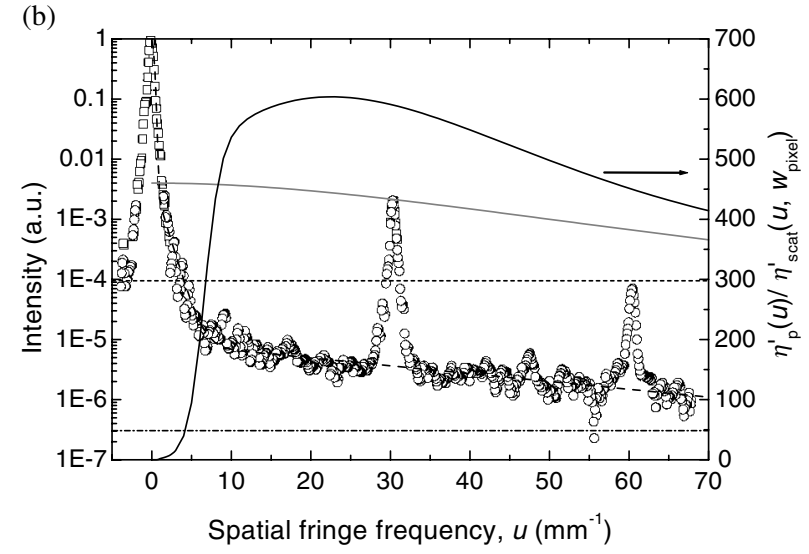

Figure 2. (a) Averaged background subtracted image of Fourier plane with $20 \mathrm{~ms}$ exposure (displayed using a logarithmic grey scale) this image corresponds to image $2 \mathrm{a}-2 \mathrm{~b}$ using the notation in the text. The white line shows where the line profile is taken.

(b) Measurement of the scattering efficiency as a function of spatial frequency: $(\square)$ points recorded with $0.1 \mathrm{~ms}$ exposure time, $(O)$ points recorded with $20 \mathrm{~ms}$ exposure, long dashed line is an empirical fit to the data; grey curve shows the output diffraction efficiency as a function of grating frequency and the black solid curve shows $\eta_{\mathrm{p}}^{\prime}(u) / \eta_{\text {scat }}^{\prime}\left(u, w_{\text {pixel }}\right)$, the ratio of the diffracted signal to the scattered signal for an effective slit width defined by the CCD pixel size (right-hand axis). Short dashed and dash-dotted lines show the rms noise levels for the 0.1 and $20 \mathrm{~ms}$ exposures respectively.

distribution in the Fourier plane $(2 a-2 b)$. For each shutter time, a line profile was taken through the background subtracted image obtained, with the line being chosen so as to pass through the central maximum of the spots formed by the diffracted beams, as shown in figure 2(a). These two curves were combined, scaled such that the peak of the zeroth order spot has a value of unity, and the results are shown in figure 2(b). The zeroth, first and second order diffraction peaks are seen above the background of scattered light, which slowly decays at higher spatial frequencies. The results in figure 2(b) are plotted as a function of spatial frequency, $u$, which in the Fourier plane is directly proportional to spatial position on the CCD. For this experiment, the fringe pattern is slightly tilted from the vertical in order to move the diffracted signal away from the diffraction pattern of the rectangular PRQW device aperture. The noise level was also measured for each CCD exposure time by measuring the root-mean square (rms) fluctuation of the line profile resulting from the differenced background images $(b-c)$.

The output diffraction efficiency (ratio of transmitted zeroth order to first order intensity) of the device was measured as a function of grating period and fitted to the following model [22]:

$$
\eta_{\mathrm{p}}^{\prime}(\Lambda)=\frac{\eta_{\mathrm{p} \max }^{\prime}}{\left[1+\left(u / u_{\mathrm{c}}\right)^{2}\right]^{2}} .
$$

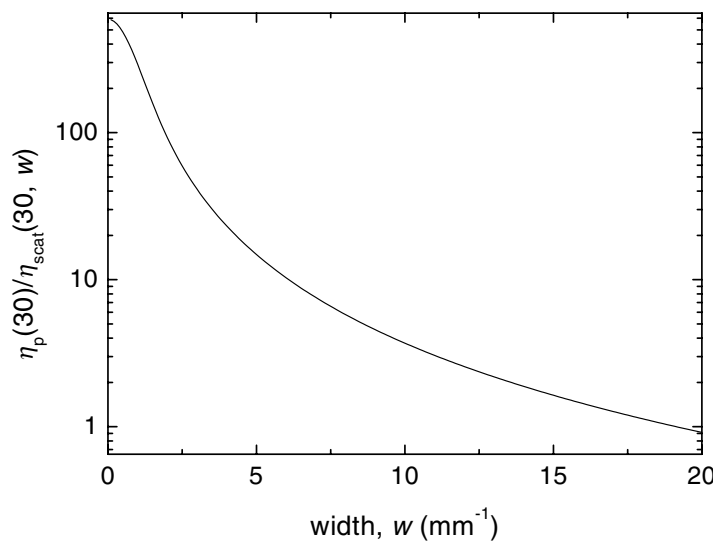

Figure 3. Plot showing variation in $\eta_{\mathrm{p}}(u) / \eta_{\text {scat }}(u, w)$ as a function of $w$, for $u=30 \mathrm{~mm}^{-1}$.

Here $\eta_{\mathrm{pmax}}^{\prime}$ is the peak output diffraction efficiency, $u_{\mathrm{c}}$ is the characteristic spatial fringe frequency for the device and the prime symbol is used to denote the output rather than the input diffraction efficiency. The output and input diffraction efficiencies differ by a factor equal to the transmission of the device at the readout beam wavelength. For our device, $1 / u_{\mathrm{c}}=\Lambda_{\mathrm{c}}$ was found to be $20 \mu \mathrm{m}$ and $\eta_{\mathrm{p} \max }^{\prime}$ equal to $4 \times 10^{-3}$. These values are sub-typical of such PRQW devices, as in general $\Lambda_{\mathrm{c}}$ can be as small as $5 \mu \mathrm{m}$ and $\eta_{\mathrm{p}}^{\prime}$ can be as high as several per cent.

Finally, the ratio of the diffraction and scattering efficiencies, $\eta_{\mathrm{p}}(u) / \eta_{\text {scat }}\left(u, w_{\text {pixel }}\right)=\eta_{\mathrm{p}}^{\prime}(u) / \eta_{\text {scat }}^{\prime}\left(u, w_{\text {pixel }}\right)$, can be calculated as a function of spatial frequency and this is also shown in figure 2 (solid black curve). Note that this curve corresponds to the case where the width of the detection aperture is defined by the CCD pixel size i.e. $w_{\text {pixel }}$. As the aperture size, and hence the achievable image resolution, is increased, the decrease in $\eta_{\mathrm{p}}(u) / \eta_{\text {scat }}(u, w)$ is approximately proportional to the area of the Fourier plane slit, i.e. $\propto 1 / w^{2}$. More accurately, the dependence on $w$ may be calculated by integrating the two-dimensional distributions of $\eta_{\mathrm{p}}$ and $\eta_{\text {scat }}$ over a square of side $w$, centred a distance $u$ from the zeroth order. This may be performed by assuming a Gaussian distribution for the diffracted signal and using an empirical exponential decay model fitted to the measured scattered light distribution (figure 2(b)) for $\eta_{\text {scat }}(u)$. Here the scattered light distribution is assumed to be rotationally symmetric about the readout beam axis. The results of this modelling are shown in figure 3 for $u=30 \mathrm{~mm}^{-1}$. Points beyond $w=20 \mathrm{~mm}^{-1}$ are not shown as an aperture above this size can allow unscattered zeroth order components to reach the CCD; $w_{\max }=2 u / 3$ [30].

We note that the values of $\eta_{\mathrm{p}}(u=30) / \eta_{\text {scat }}(u=$ $30, w)$ shown in figure 3 are smaller than that previously measured [24], partly because this particular PRQW device exhibits a significantly lower diffraction efficiency than that used for our previous measurement and partly because the previous value was obtained by measuring the scattered light at an image plane and over a particular area of the PRQW that was free of any large (visible) defects. This is reasonable because these large defects result from air bubbles trapped in the epoxy and should be straightforward to eliminate by performing the fabrication under clean-room conditions. The present Fourier plane measurements probe the angular 
scattered light distribution from the whole PRQW aperture and so are sensitive to any large defects. While they represent a 'worst case scenario', the measurements presented here provide a straightforward means to compare the diffracted and scattered signals and investigate the trade-off between signal to noise ratio and lateral resolution. Figure 2(b) shows that the maximum value of $\eta_{\mathrm{p}}(u) / \eta_{\text {scat }}\left(u, w_{\text {pixel }}\right)$ obtained for this device occurs at a spatial fringe frequency of $\sim 23 \mathrm{~mm}^{-1}$ and therefore this is the optimum spatial fringe frequency to maximize the ratio of signal to background noise. This optimum fringe frequency is independent of the size of the Fourier plane aperture although the peak value of $\eta_{\mathrm{p}}(u) / \eta_{\text {scat }}(u, w)$ will decrease as $w$ is increased. In general it is necessary to select $u$ and $w$ in order to optimize the set-up for a given experimental objective. For example if achieving a high diffraction efficiency is the most important goal, then it may be necessary to sacrifice some spatial resolution and dynamic range by operating at slightly smaller fringe spatial frequencies.

Similar considerations will apply to other photorefractive media although the optimum trade-offs may result in different optimum fringe spatial frequencies. For example, a sample of photorefractive $45^{\circ}$-cut rhodium-doped barium titanate is optimally deployed with a fringe period of $\sim 1 \mu \mathrm{m}$ in an image plane geometry. For incident intensities of $\sim 20 \mathrm{~mW} \mathrm{~cm}^{-2}$, the output diffraction efficiency achieved with this crystal reached $25 \%$ for exposure times of $\sim 30 \mathrm{~s}$. For this particular crystal $\eta_{\mathrm{p}} / \eta_{\text {scat }}$ was found to be $\sim 5$ for a detector solid angle was $3.1 \times 10^{-4}$ sr. The main reason that $\eta_{\mathrm{p}} / \eta_{\text {scat }}$ was so low was due to a diagonal defect across the crystal that could not be completely avoided whilst making this measurement. For crystals with no visible defects, we would expect $\eta_{\mathrm{p}} / \eta_{\text {scat }}$ to be significantly greater.

\section{High-frame-rate coherence gated imaging}

A major advantage of PRQW devices is their extremely rapid response times and so using them for photorefractive holography can provide very high-frame-rate coherence-gated imaging. Compared to digital holography, for which the CCD must adequately sample the hologram fringe pattern, photorefractive holography requires only that the diffracted image (rather than the fringe pattern) is adequately sampled, thus reducing the resolution requirements of the CCD. Compared to phase-stepping holography, for which the fringe pattern must be effectively stationary on the timescale of the multiple CCD exposures, photorefractive holography requires that it is stationary only on the time scale of the grating formation process. Balasubramanian et al [31] have measured the grating recording time (time taken for diffracted signal to reach $(1-1 / e)$ of the steady state value) to be smaller than $10 \mu \mathrm{s}$ for an incident intensity of $10 \mathrm{~mW} \mathrm{~cm}^{-2}$ and this corresponds to an imaging rate of greater than $100 \mathrm{kHz}$. Previously, imaging with PRQW devices has achieved a frame rate of $470 \mathrm{fps}$ using an intensified readout camera [32]. We now report a depth-resolved imaging rate of $830 \mathrm{fps}$ using lowcoherence photorefractive holography with a PRQW device.

In order to demonstrate high-frame-rate photorefractive holography, the readout camera was replaced with a Dalsa CAD1 8-bit CCD camera running at $830 \mathrm{fps}$, with an exposure time (a)
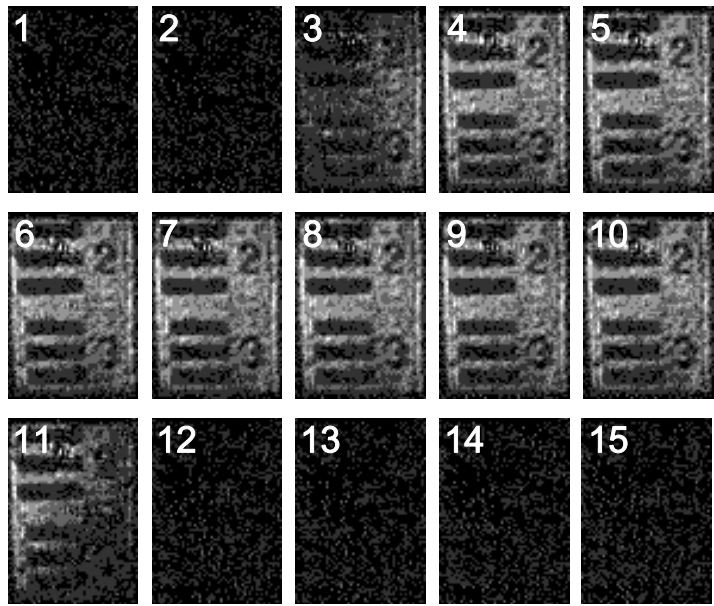

(b)

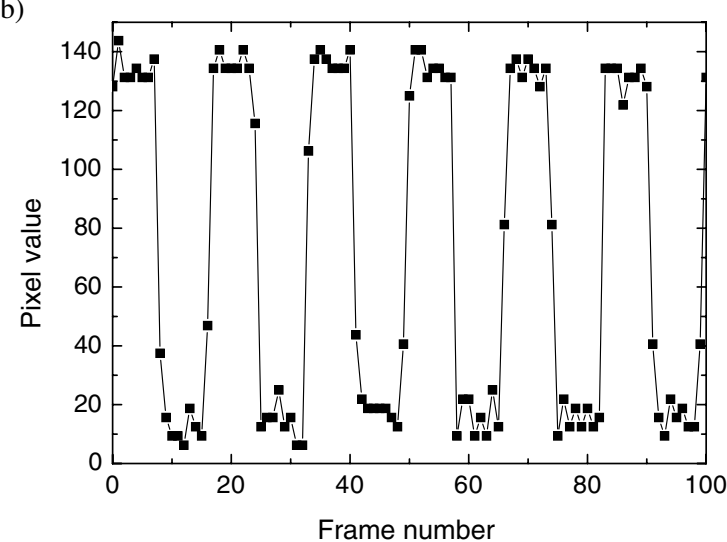

Figure 4. (a) Consecutive images of test chart acquired at $830 \mathrm{fps}$ with a chopper blade rotating at $50 \mathrm{~Hz}$. Region shown is only part of the whole acquired image and has an area of $56 \times 78$ pixels. The corresponding field of view is $2 \mathrm{~mm} \times 2.8 \mathrm{~mm}$. (b) Plot of the pixel values averaged across a $4 \times 4$ pixel area for 100 frames $(120 \mathrm{~ms})$.

of $1 \mathrm{~ms}$ and an image size of $128 \times 128$ pixels. To provide a simple dynamic object, an optical chopper wheel running at $50 \mathrm{~Hz}$ was placed in front of a USAF test chart (see dashed line in object arm, figure 1). A series of images were acquired at $830 \mathrm{fps}$ and these images are shown in figure 4(a). The only post-processing step was to subtract a fixed background image from each of the frames to remove the bright PRQW scattering centres. The write and readout beam intensities at the PRQW device were both $300 \mu \mathrm{W} \mathrm{cm}{ }^{-2}$ and a fringe period of $37 \mu \mathrm{m}$ was used. The test chart bars are group 1 and the element 2 bars seen in figure 4(a) have a width of $0.25 \mathrm{~mm}$. The chopper blade can clearly be seen obscuring the test chart, moving (at a slight angle to the horizontal) from right to left. The chopper blade velocity across the field of view was calculated to be $2.8 \mathrm{~m} \mathrm{~s}^{-1}$, therefore during the $\mathrm{CCD}$ integration time of $1 \mathrm{~ms}$ this corresponds to a movement of $2.8 \mathrm{~mm}$ which is roughly equivalent to the field of view $(2 \mathrm{~mm} \times 2.8 \mathrm{~mm})$. This movement compares well with that seen in figure 4(a) and suggests that it is the CCD exposure time, rather than the PRQW response time that prevents the edge of the blade shadow from being clearly resolved in this experiment. Figure 4(b) shows the temporal evolution of the signal measured from a $4 \times 4$ pixel area taken from the centre 
of the readout image. The $50 \mathrm{~Hz}$ oscillation can be clearly seen and this graph enabled the achieved frame rate to be verified.

\section{Conclusions}

Photorefractive holography is a method for performing widefield coherence gated imaging. When implemented using PRQW devices, high-speed depth-resolved imaging (optical sectioning) is possible, with frame rates of $830 \mathrm{fps}$ having been demonstrated. In common with other wide-field coherence gating techniques, photorefractive holography can exploit lowcost broadband sources with low spatial coherence. Unlike other coherence-gated imaging approaches, photorefractive holography provides a means to remove a diffuse scattered light background although we note that its sensitivity and dynamic range are subject to trade-offs that do not apply to techniques relying on direct detection at a CCD. To investigate these tradeoffs, we have presented here an experimental procedure that may be used to characterize the performance of photorefractive holographic recording media by measuring the Fourier plane distribution of scattered light that arises from imperfections in the PRQW over a large dynamic range using a standard video CCD camera. This measurement allows the output scattering efficiency, $\eta_{\text {scat }}^{\prime}$, to be directly compared to the output diffraction efficiency, $\eta_{\mathrm{p}}^{\prime}$, of the device and hence allows the ratio of these two quantities to be measured as a function of spatial frequency in the Fourier plane. This ratio is a useful parameter in determining the ability of the system to measure weak diffracted signals although, since it is a function of the scattered light distribution averaged over the entire PRQW device aperture, it should be considered as a 'worst case' estimate. In these experiments $\eta_{\text {scat }}^{\prime}$ is dominated by the large visible defects arising from bubbles in the epoxy, which should be straightforward to eliminate with improved fabrication techniques. To the best of our knowledge, the widefield coherence gated imaging system operating at $830 \mathrm{fps}$ with a PRQW device is the fastest such system yet reported. In the near future we plan to investigate the use of an intensified high frame-rate readout CCD camera to increase the system sensitivity and aim to realize volumetric imaging using a rapidly scanning reference arm delay line.

\section{Acknowledgments}

CD acknowledges a CASE QUOTA studentship from the Engineering and Physical Sciences Council (EPSRC) and Holoscan UK Ltd. DM-C acknowledges funding from the Consejo Nacional para la Ciencia y Tecnologia and the Universidad Autonoma del Estado de Morelos and IM acknowledges funding from a DTI Beacon Award.

\section{References}

[1] Dunsby C and French P M W 2003 Techniques for depth-resolved imaging through turbid media including coherence-gated imaging J. Phys. D: Appl. Phys. 36 R207-27

[2] Huang D, Swanson E A, Lin C P, Schuman J S, Stinson W G, Chang W, Hee M R, Flotte T, Gregory K, Puliafito C A and Fujimoto J G 1991 Optical coherence tomography Science $2541178-81$
[3] Rollins A M, Kulkarni M D, Yazdanfar S, Ung-arunyawee R and Izatt J A 1998 In vivo video rate optical coherence tomography Opt. Express 3 219-29

[4] Vabre L, Dubois A and Boccara A C 2002 Thermal-light full-field optical coherence tomography Opt. Lett. 27 530-2

[5] Bordenave E, Abraham E, Jonusauskas G, Tsurumachi N, Oberle J, Rulliere C, Minot P E, Lassegues M and Bazeille J E S 2002 Wide-field optical coherence tomography: imaging of biological tissues Appl. Opt. 41 2059-64

[6] Beaurepaire E, Boccara A C, Lebec M, Blanchot L and Saint-Jalmes H 1998 Full-field optical coherence microscopy Opt. Lett. 23 244-6

[7] Dunsby C, Gu Y and French P M W 2003 Single-shot phase-stepped wide-field coherence-gated imaging $O p t$ Express 11 105-15

[8] Ducros M, Laubscher M, Karamata B, Bourquin S, Lasser T and Salathe R P 2002 Parallel optical coherence tomography in scattering samples using a two-dimensional smart-pixel detector array Opt. Commun. 202 29-35

[9] Fercher A F, Hitzenberger C K, Kamp G and Elzaiat S Y 1995 Measurement of intraocular distances by backscattering spectral interferometry Opt. Commun. 117 43-8

[10] Zuluaga A F and Richards-Kortum R 1999 Spatially resolved spectral interferometry for determination of subsurface structure Opt. Lett. 24 519-21

[11] Stetson K A 1967 Holographic fog penetration J. Opt. Soc. Am. 57 1060-1

[12] Caulfield H J 1968 Holographic imaging through scatterers J. Opt. Soc. Am. 58 276-7

[13] Chen H, Chen Y, Dilworth D, Leith E, Lopez J and Valdmanis J 1991 Two-dimensional imaging through diffusing media using 150 fs gated electronic holography techniques Opt. Lett. 16 487-9

[14] Cuche E, Marquet P and Depeursinge C 1999 Simultaneous amplitude-contrast and quantitative phase-contrast microscopy by numerical reconstruction of Fresnel off-axis holograms Appl. Opt. 38 6994-7001

[15] Mamaev A V, Ivleva L L, Polozkov N M and Shkunov V V 1993 Photorefractive visualisation through opaque scattering media: Paper CFK6 Conf. on Lasers and Electro-Optics vol 11 pp 632-3

[16] Hyde S C W, Barry N P, Jones R, Dainty J C and French P M W 1995 Sub-100 $\mu \mathrm{m}$ Depth-resolved holographic imaging through scattering media in the near-infrared Opt. Lett. 20 2330-2

[17] Jones R, Hyde S C W, Lynn M J, Barry N P, Dainty J C, French P M W, Kwolek K M, Nolte D D and Melloch M R 1996 Holographic storage and high background imaging using photorefractive multiple quantum wells Appl. Phys. Lett. 69 1837-9

[18] Tziraki M, Jones R, French P M W, Melloch M R and Nolte D D 2000 Photorefractive holography for imaging through turbid media using low coherence light Appl. Phys. B 70 151-4

[19] Sun P C and Leith E N 1994 Broad-source image plane holography as a confocal imaging process Appl. Opt. 33 597-602

[20] Barry N P, Jones R, Hyde S C W, Dainty J C and French P M W 1997 High background holographic imaging using photorefractive barium titanate Electron. Lett. $\mathbf{3 3}$ 1732-3

[21] Wang Q, Brubaker R M, Nolte D D and Melloch M R 1992 Photorefractive quantum-wells - transverse Franz-Keldysh geometry J. Opt. Soc. Am. B 9 1626-41

[22] Nolte D D and Melloch M R 1995 Photorefractive quantum wells and thin films Photorefractive Effects and Materials ed D D Nolte (Dordrecht: Kluwer) pp 373-452

[23] Yu P, Peng L L, Mustata M, Nolte D D, Turek J J, Melloch M R, Dunsby C, Gu Y and French P M W 2003 Imaging of tumor necroses using full-frame optical coherence imaging Coherence Domain Optical Methods 
and Optical Coherence Tomography in Biomedicine VII (Proc. SPIE vol 4956) (Bellingham, WA: SPIE Optical Engineering Press)

[24] Dunsby C, Gu Y, Ansari Z, French P M W, Peng L, Yu P, Melloch M R and Nolte D D 2003 High-speed depth-sectioned wide-field imaging using low-coherence photorefractive holographic microscopy Opt. Commun. 219 87-99

[25] Dunsby C, Gu Y, Ansari Z, Parsons-Karavasillis D, Siegel J, Itoh M, Iwamoto S, Headley W, Melloch M R, Nolte D D and French P M W 2002 Low coherence photorefractive holography for high-speed 3D imaging, including through scattering media Coherence Domain Optical Methods in Biomedical Science and Clinical Applications VI (Proc. SPIE vol 4619) (Bellingham, WA: SPIE Optical Engineering Press) pp 98-106

[26] Parsons-Karavassilis D, Gu Y, Ansari Z, French P M W and Taylor J R 2000 Diode-pumped spatially dispersed broadband Cr:LiSGAF and Cr:LiSAF cw laser sources applied to short-coherence photorefractive holography $O p t$. Commun. 181 361-7
[27] Leith E N and Swanson G J 1980 Achromatic interferometers for white light optical processing and holography Appl. Opt. $19638-44$

[28] Chmelik R and Harna Z 1999 Parallel-mode confocal microscope Opt. Eng. 38 1635-9

[29] Ansari Z, Gu Y, Tziraki M, Jones R, French P M W, Nolte D D and Melloch M R 2001 Elimination of beam walk-off in low-coherence off-axis photorefractive holography Opt. Lett. 26 334-6

[30] Hariharan P 1996 Optical Holography (Cambridge: Cambridge University Press)

[31] Balasubramanian S, Lahiri I, Ding Y, Melloch M R and Nolte D D 1999 Two-wave-mixing dynamics and nonlinear hot-electron transport in transverse-geometry photorefractive quantum wells studied by moving gratings Appl. Phys. B 68 863-9

[32] Gu Y, Ansari Z, Dunsby C, Parsons-Karavassilis D, Siegel J, Itoh M, French P M W, Nolte D D, Headley W and Melloch M R 2002 High-speed 3D imaging using photorefractive holography with novel low-coherence interferometers J. Mod. Opt. 49 877-87 